\title{
Telemonitoring von Armergometer-Training bei COPD-Patienten. Eine Pilotstudie
}

\author{
Telemonitoring of Arm Ergometric Training in COPD Patients. A Pilot Study
}

Autoren

Institut
K. H. Rühle, M. Bloch, K. J. Franke, G. Nilius

HELIOS-Klinik Hagen-Ambrock, Universität Witten/Herdecke eingereicht 13.11.2008 akzeptiert nach Revision 30.1.2009

\section{Bibliografie}

DOI $10.1055 / \mathrm{s}-0028-1119701$

Online-Publikation: 16. 3. 2009

Pneumologie 2009; 63:

314-318 @ Georg Thieme

Verlag KG Stuttgart · New York ISSN 0934-8387

Korrespondenzadresse Prof. Dr. med. Karl-Heinz Rühle HELIOS-Klinik Hagen-Ambrock Klinik für Pneumologie 58091 Hagen

Klinik-Ambrock.Pneumo@ t-online.de

\section{Zusammenfassung \\ $\nabla$}

Einleitung: Regelmäßige moderate körperliche Aktivität bei COPD führt zu einer Verringerung der Morbidität und Verbesserung der Lebenserwartung. Viele Programme scheitern oftmals daran, dass nach Rehabilitation ein regelmäßiges Training zu Hause, evtl. durch mangelnde Motivation bedingt, nicht durchgeführt wird; die Leistungsparameter fallen relativ schnell auf den Ausgangswert zurück. Aus Kostengründen wird ein einfaches Verfahren zur Messung der Trainingszeiten für das häusliche Umfeld mit täglicher telemetrischer Übertragung der Daten gesucht, sodass bei Veränderung der Trainingsleistung motivierend eingegriffen werden kann. Fragestellung: Wie lange dauert bei einer Vorgabe von 20 Minuten/Tag durch den Arzt die tatsächliche tägliche Trainingszeit der Patienten? Existiert ein Zusammenhang zwischen körperlicher Aktivität zu Hause und der telemetrisch erfassten Trainingsdauer? Methodik: Eingesetzt wurde ein Gerät zum Training der oberen Extremitäten mit einem GSM-Modul zur Datenfernübertragung der Trainingszeiten. Die körperliche Aktivität vor einer Exacerbation der COPD und die Bereitschaft zur Veränderung des Trainingsverhaltens wurden mit Fragebögen erfasst. 13 Patienten mit COPD (Alter $69 \pm 9$ Jahre, FEV1 48,6 $\pm 23,9 \%$ Soll) wurden prospektiv über 6 Tage unter stationären Bedingungen untersucht. Ergebnisse: Die mittlere Trainingsdauer/Tag (Vorgabe 20 Minuten/Tag) lag bei 16,1 \pm 11 ,7 Minuten/ Tag. Es zeigte sich eine signifikante Korrelation zwischen der Frequenz der körperlichen Aktivität/ Woche und der täglichen Ergometrie-Trainingsdauer in der Klinik $(r=0,59)$. Schlussfolgerung: Telemonitorisch betreutes Training stellt eine praktikable Methode dar, um objektiv Trainingszeiten zu erfassen. Häusliches Ergometer-Training in der Kombination mit Online-Übertragung (Telemonitoring) und regelmäßiger Telefonbetreuung könnte ein kostengünstiges Verfahren zur Erhöhung der körperlichen Aktivität darstellen.

\section{Abstract \\ $\nabla$}

Introduction: Regular moderate physical activity in COPD reduces morbidity and improves life expectancy. Many programmes often fail after rehabilitation because regular training at home, possibly caused by a lack of motivation, is not maintained, the performance parameters then decline to the prerehabilitation levels. For cost reasons, a simple procedure is required to measure the training times in the home environment with a daily transmission of telemetric data so that, in the case of decline, advice and assistance can be provided. Questions: How long is the actual daily training time monitored by telemetry of COPD patients if a target of 20 minutes per day is prescribed by the doctor? Is there a correlation between physical activity at home and the training period? Method: We used a device for training the upper extremities with a GSM data transmission module for the transfer of the training times. The physical activity before an exacerbation of COPD and the willingness to modify the training behaviour were determined by questionnaires. 13 patients with COPD (age $69 \pm 9$ years, FEV1 $48.6 \pm 23.9 \%$ ) were investigated prospectively for 6 days under stationary conditions. Results: The mean training period (target $20 \mathrm{~min} /$ day) was $16.1 \pm 11.7 \mathrm{~min} /$ day. The frequency of physical activity per week correlated with the daily ergometry training period in the clinic $(r=0.59)$. Conclusion: Training supervised by telemonitoring represents a useful way to objectively obtain training times. Ergometric training in combination with online transmission (telemetric monitoring) and regular phone calls could be an inexpensive method to increase physical activity. 


\section{Einleitung}

\section{$\nabla$}

Ergometertraining hat den Vorteil, dass die physikalische Leistung genau dosiert und unter gleichen Bedingungen reproduziert werden kann. Der Trainingsumfang ist genau definiert, sodass die tägliche Leistung messbar wird.

Im Rahmen eines ambulanten, achtwöchigen Trainingsprogramms, das zweimal in der Woche in einem Trainingszentrum stattfand und unter anderem ein Ausdauertraining auf dem Fahrrad-Ergometer beinhaltete, konnte bei COPD-Patienten eine deutlich verbesserte Ausdauerleistung und eine Verlängerung der 6-Minuten Gehstrecke erzielt werden [1].

Gemeinsames Training im Sportgruppen ist wegen der Kosten und langen Anfahrtszeiten häufig nicht möglich oder zu aufwendig.

Ergometertraining eignet sich besonders für den Einsatz zu Hause, da dieses vom Wetter und den klimatischen Bedingungen unabhängig ist. Auch steht es permanent zur Verfügung und ist unabhängig von dem Zugang zu Übungsräumen oder Sporthallen. Aber viele Trainingsprogramme scheitern oftmals, da ein regelmäßiges Training zu Hause, evtl. durch mangelnde Motivation bedingt, nicht durchgeführt wird.

Wird das Training allerdings ausgesetzt und finden keine Interventionen statt, fallen die Leistungsparameter relativ schnell wieder auf den Ausgangswert zurück [2].

Es sind deshalb Konzepte erforderlich, die langfristig die erreichte Leistungssteigerung durch die initiale Rehabilitation erhalten beziehungsweise noch ausbauen. Dies gilt umso mehr, weil nur eine regelmäßige zumindest moderate körperliche Aktivität zu einer Verringerung der Morbidität und Verbesserung der Lebenserwartung führt [3]. Wichtig ist soziale Unterstützung, um ein tägliches körperliches Training aufrechtzuerhalten. Dies kann unter anderem durch telefonischen Kontakt des betreuenden Rehabilitationsarztes mit dem Patienten zur Erhöhung der Motivation erfolgen. So konnte durch regelmäßige Telefongespräche, verglichen mit den Basisdaten bei COPD-Patienten die 6-Minuten-Gehstrecke und die körperliche Aktivität gesteigert werden [4].

Hilfreich wäre es, wenn für diese Gespräche die aktuelle, gemessene Trainingszeit und Trainingsleistung zur Verfügung stünde. Einige für das häusliche Training geeignete Ergometer besitzen bereits eine Registriermöglichkeit, wobei die täglichen Trainings-Zeiten mittels Flash-Card aufgezeichnet werden [5].

Für den routinemäßigen Einsatz mit Verschickung der Karte durch die Post dürfte dieses Verfahren aber nur bei längerfristigen Intervallen praktikabel sein.

Wünschenswert wäre deshalb, dass ein einfaches Verfahren zur Messung der Trainingszeiten mit täglicher telemetrischer Übertragung der Daten zur Verfügung steht. Wir rüsteten deshalb in einem ersten Schritt ein Gerät zum Training der unteren und oberen Extremitäten mit einem GSM-Modul zur Datenfernübertragung der Trainingszeiten aus. Mit jeweils einem Fragebogen wurde die körperliche Aktivität vor der Exazerbation der COPD und die Bereitschaft zur Veränderung des Trainingsverhaltens erfasst [6, 7].

Fragestellung: In der vorliegenden Pilotstudie sollte untersucht werden, inwiefern Patienten nach akuter Exazerbation ihrer COPD zu einem täglichen Training der oberen Extremitäten von $2 \times 10$ Minuten bereit bzw. in der Lage sind.

Unsere Fragestellung lautete deshalb: Wie hoch liegt bei einer Vorgabe von $2 \times 10$ Minuten/Tag durch den Arzt die tatsächliche tägliche Trainingszeit der Patienten?

Existiert ein Zusammenhang zwischen körperlicher Aktivität in der Freizeit während des Zeitraums einer Woche, die am Tage

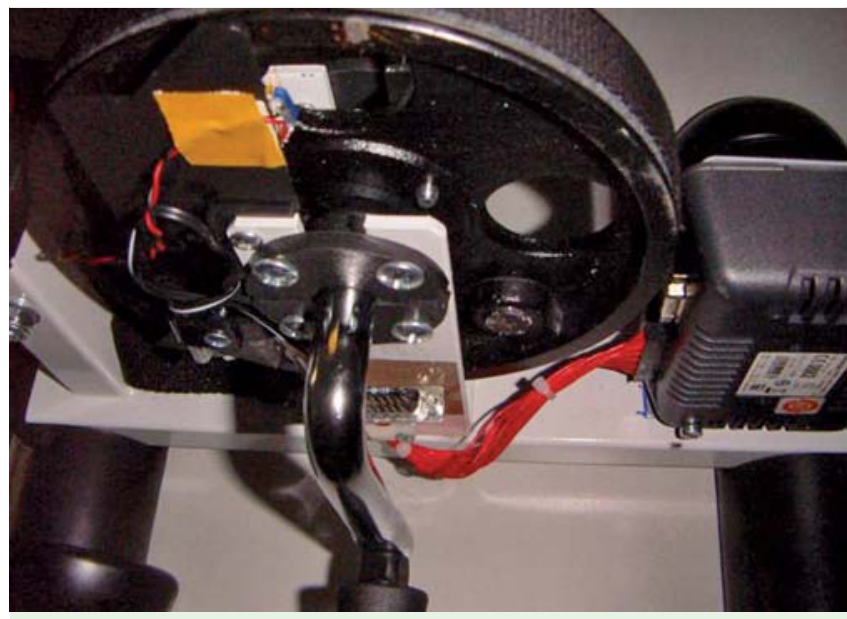

Abb. 1 Bei einer Drehbewegung der Pedale wird durch den Magnetschalter am Schwungrad ein Schaltimpuls ausgelöst, der durch die Auswertelogik in einem Mikro-Controller (GSM-Modul TC-65 Firma Siemens) verarbeitet wird. 15 Sekunden nach Trainingsbeginn werden die Trainingssekunden gezählt. Werden 15 Sekunden lang keine Impulse registriert, erfolgt die Trainingszeit-Berechnung. Die Daten werden per SMS verschickt. Es können bis zu 8 Input-/Output-Kanäle angeschlossen werden.

länger als 20 Minuten beträgt, und der telemetrisch erfassten Trainingsdauer.

\section{Methodik \\ $\nabla$}

Die Studie wurde von der Ethik-Kommission der Universität Witten-Herdecke geprüft und genehmigt.

Patienten: 13 Patienten mit COPD (5 Frauen und 8 Männer, mittleres Alter $69 \pm 9$ Jahre, Sekundenkapazität 48,6 $\pm 23,9 \%$ Soll) wurden prospektiv untersucht.

Einschlusskriterien: Patienten der Klinik für Pneumologie mit lungenfunktionell gesicherter COPD im stabilen Zustand, GoldStadium 3-4 im Alter zwischen 40 und 80 Jahren wurden ganzkörperplethysmographisch untersucht und über das geplante Trainingsprogramm durch die Physiotherapeutin informiert sowie in einem 20-minütigen Arztgespräch motiviert, mindestens $2 \times 10 \mathrm{~min} /$ Tag ein Armergometertraining durchzuführen. Nach schriftlichem Einverständnis erhielten die Patienten ein Handkurbel-Ergometer, das im Krankenzimmer des Patienten aufgestellt wurde. Die Trainingszeit wurde über 6 Tage hinweg telemonitorisch erfasst.

Die Patienten erhielten 2 Fragebögen. Gefragt wurde anhand eines Fragebogens zum Trainingsverhalten vor der Exazerbation nach dem Schweregrad der Belastung und wöchentlichen Frequenz einer $>20 \mathrm{~min} /$ Tag dauernden körperlichen Belastung sowie der Häufigkeit von körperlichen Aktivitäten, die zu Schwitzen und schnellerem Herzschlag führen - (Godin Leisure Time Exercise Questionnaire, GLTEQ Godin) [6] - und nach dem Stadium der Absicht zur Veränderung, z. B.: Ich beabsichtige in den nächsten 30 Tagen/6 Monaten/körperliches Training/kein Training (Kurzform) [7].

Ein handelsübliches Handkurbel-Ergometer (BE Bodyfit Minitrimmer Meteor 100, Fa Stamm Gummersbach) wurde mit einem Datenerfassungssystem GSM-Modul TC-65 mit integriertem Mikrokontroller (Firma Siemens) zur automatischen Registrierung der Trainingszeiten ausgerüstet $(\bullet$ Abb. 1). 


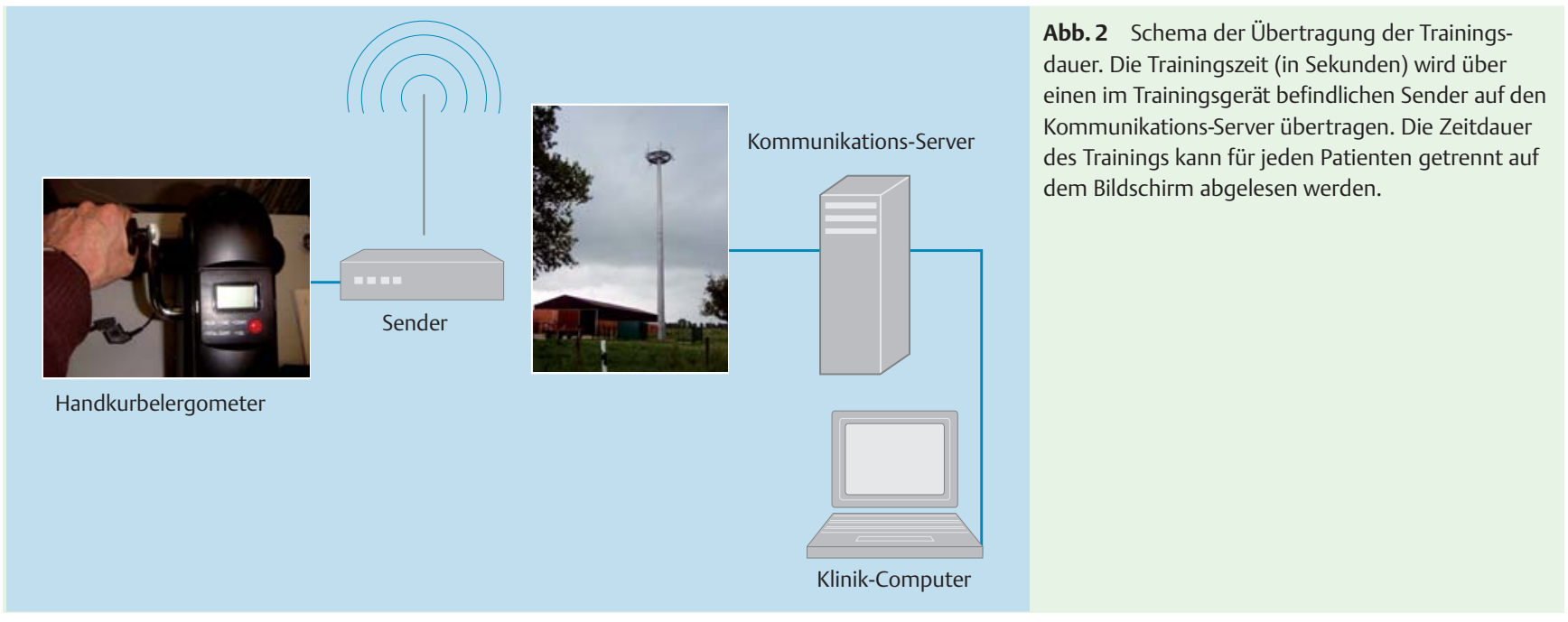

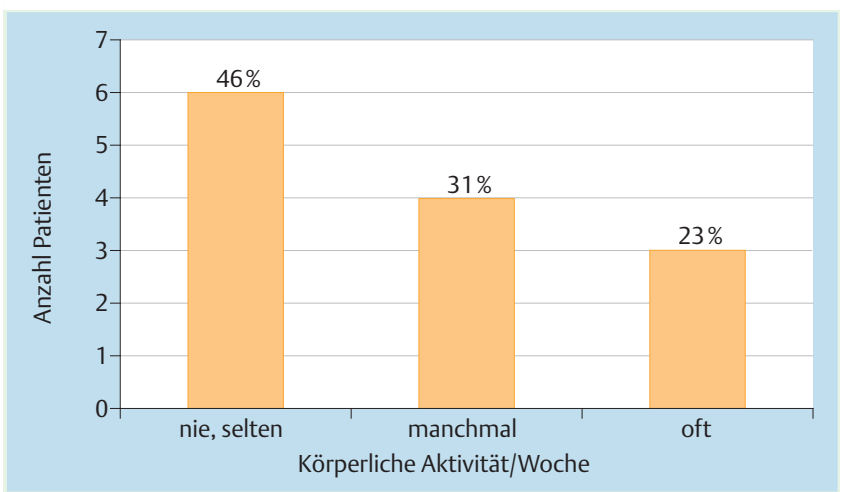

Abb. 3 Prozentuale Verteilung der Antworten der 13 COPD-Patienten auf die Frage: Wenn Sie den Zeitraum einer Woche betrachten, wie oft entwickeln Sie eine körperliche Aktivität, die zu Schwitzen und schnellerem Herzschlag führt? Die Frage bezieht sich auf den Zeitraum vor der akuten Exazerbation.

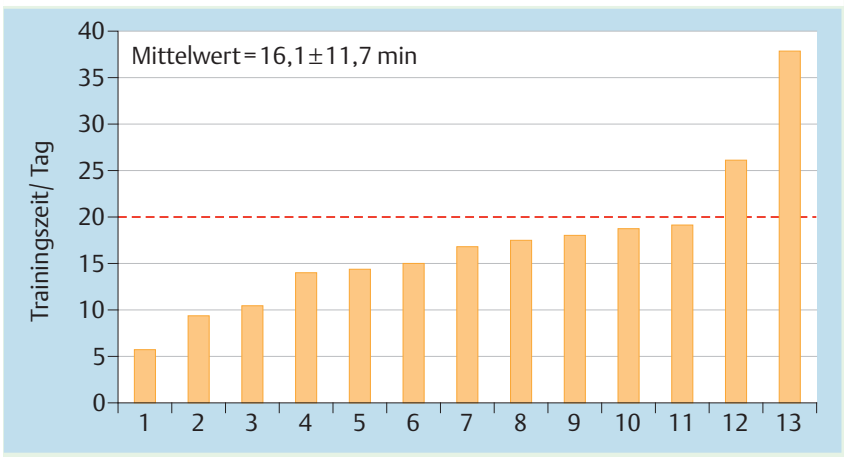

Abb. 4 Verteilung der durchschnittlichen Trainingszeit/Tag bei 13 COPDPatienten. Die gepunktete Linie zeigt das Soll der Trainingszeit, das nur von 2 Patienten erreicht wird.

Bei Beginn der ersten Umdrehung wird die Zeit bis zum Ende der Tätigkeit automatisch erfasst. Finden innerhalb von 15 Sekunden keine Umdrehungen statt, stoppt die Registrierung. Der Impuls zur Registrierung einer Umdrehung wird über einen berührungslosen Magnetschalter erzeugt. Es besteht keinerlei elektrischer Kontakt zwischen Gerät und Patient. Die Daten werden codiert per GPRS an einen Klinik-Server versandt ( $\bullet$ Abb. 2).

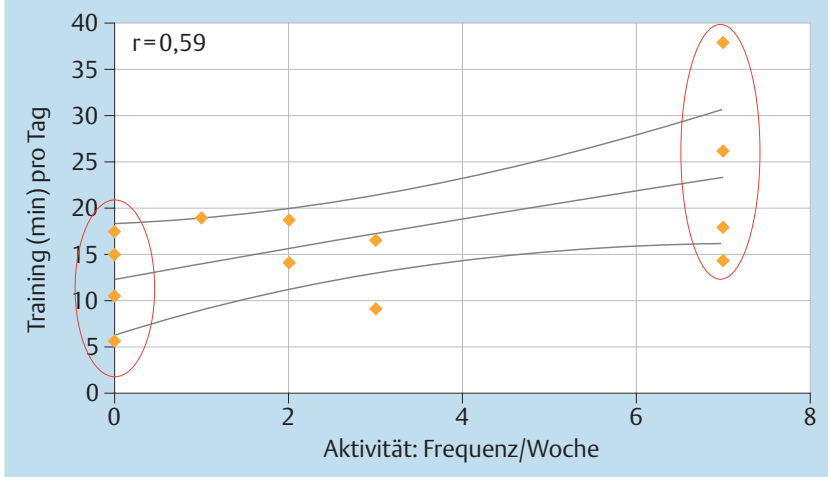

Abb. 5 Korrelation zwischen der häuslichen Aktivität (physical activity, GLTEQ) und der individuellen telemetrisch erfassten Trainingszeit von 13 Patienten mit COPD. Die höheren Trainingszeiten wurden von denjenigen Patienten erreicht, die regelmäßige, mehr als 20 Minuten dauernde körperliche Bewegung angaben.

Die Übertragung der Daten erfolgt über SSL-Verschlüsselung. Es wird nur die anonyme Patienten-Identifikation (ID) übertragen. Nur der Prüfungsleiter hat Zugriff über das Web-Frontend. Die Datenbank ist von außen ohne die Anwendung nicht erreichbar. Die Daten sind nur für den Arzt über ein Passwort erreichbar, sodass nur er die täglichen Trainingszeiten einsehen kann.

\section{Ergebnisse}

Bei der Frage nach der Häufigkeit von körperlichen Aktivitäten, die zu Schwitzen und schnellerem Herzschlag führen, gab fast die Hälfte der Patienten selten oder nie an. Nur etwa ein Viertel der Patienten entwickelt oft die Symptome einer anstrengenden Belastung (siehe Abb. 3).

Zum Trainingsverhalten zu Hause gaben die Patienten im GLTEQ eine leichtgradige Belastung mit einer Frequenz von 3,0 \pm ,9mal/Woche $>20$ Minuten/Tag an.

Die mittlere Trainingsdauer/Tag (Vorgabe 20 Minuten/Tag) lag bei 16,1 $\pm 11,7$ Minuten/Tag. Nur 2 der Patienten überschritten die vorgegebene Trainingszeit von $20 \mathrm{~min}$ (siehe Abb. 4).

Es zeigte sich eine signifikante Korrelation zwischen der Frequenz der körperlichen Aktivität bzw. des Trainings pro Woche und der täglichen Ergometrie-Trainingsdauer in der Klinik $(r=0,59)$ (siehe $\bullet$ Abb. 5). 
Die Patienten wurden zur Trainingsabsicht nach Entlassung befragt: 5/13 der Patienten (38\%) waren nicht bereit zu trainieren. Weitere 5/13 (38\%) beabsichtigten innerhalb der nächsten 30 Tage regelmäßiges körperliches Training.

\section{Diskussion}

$\nabla$

\section{Geräteentwicklung}

Die von uns entwickelte Koppelung eines Trainingsgerätes mit einer Funkübertragung der Trainingszeiten stellt eine einfache und relativ kostengünstige Lösung dar. Die Übermittlung der Daten ist ohne Mitarbeit des Patienten möglich. Die bis jetzt praktizierten Lösungen erfordern die Kooperation des Patienten: Das Verschicken von Flash-Cards ist umständlich und nicht zeitnah. Werden die Daten über das Internet verschickt, muss eine Kabelverbindung zum Modem und Computer hergestellt werden, häufig steht ein Internetzugang nicht zur Verfügung; Übertragung durch Blue-Tooth-Verbindung setzt laufenden Computerbetrieb voraus.

In dieser Pilotstudie beabsichtigten wir, den Nachweis der Funktionstüchtigkeit und Praktikabilität des Konzeptes zu erbringen. Deshalb setzten wir das Gerät nur für das Armtraining ein. Ein Armtrainingsprogramm bei COPD kann die dynamische Überblähung, die Atemfrequenz und Dyspnoe bei Armtätigkeiten herabsetzen und erhöht die Ausdauer [8]. Deshalb wird in den Leitlinien des ACCP empfohlen, ein Training der oberen Extremitäten in einem Trainingsprogramm von COPD-Patienten einzuschließen [9].

\section{Ergebnisse}

Anhand der objektiven Messung mittels Telemonitoring konnten wir zeigen, dass $85 \%$ der Patienten die vorgegebene Trainingsdauer von 20 Minuten/Tag nicht erreichten. Es besteht damit ein hohes Potential an Verbesserungsmöglichkeiten des Trainingsverhaltens. Die Ursachen der fehlenden Mitarbeit sind vielschichtig. Die Trainingszeit der Patienten kann durch fehlende Einsicht, mangelnde Motivation, aber auch Dyspnoe oder Gelenkschmerzen und Begleiterkrankungen reduziert sein. Die Ergebnisse stimmen mit den Cochrane-Daten zusammengefasster Untersuchungen zu Interventionen zur Steigerung der körperlichen Aktivität überein, nach denen eine mäßige Steigerung, aber nicht das vorgegebene zeitliche Niveau erreicht wird [10]. Mit unseren Daten vergleichbare Ergebnisse für die Trainingszeit fanden sich in einer Studie zum häuslichen Bewegungstraining mit einem Sitzergometer bei Patienten nach Apoplexie [5]. Bei einer Vorgabe von 20 min Training/Tag lag die tatsächliche, mit einer Chipkarte aufgezeichnete durchschnittliche Trainingsdauer bei 15,4 $\pm 0,5$ Minuten.

Die körperliche Aktivität der Patienten zu Hause mit individuell höhergradigen Belastungen mit Schwitzen und hoher Pulsfrequenz war erstaunlich gering. Fast 50\% der Patienten vermeiden demnach Belastungsspitzen. Die Patienten praktizieren zu Hause nur leichte körperliche Freizeitaktivitäten $>20 \mathrm{~min} /$ Tag mit geringer Anstrengung wie z.B. ruhiges Gehen oder Nordic walking. Zwischen der Frequenz dieser körperlichen Aktivität pro Woche (f/Wo) und der täglichen Ergometrie-Trainingsdauer in der Klinik bestand ein mittlerer korrelativer Zusammenhang ( $r=0,59)$, zwischen FEV1 und f/Wo dagegen nur ein geringe Korrelation $(\mathrm{r}=0,39)$. Diese Daten passen zu der Vorstellung, dass die Trainingsaktivität neben der Atemmechanik durch weitere Faktoren wie Einstellung und Motivation beeinflusst wird. Zukünftig sollte geklärt werden, ob mit der Trainingszeit im stationären Bereich die häusliche Trainingsdauer vorhergesagt werden kann.

Welche Patienten kommen für ein telemetrisch überwachtes Training in Frage? Bevor durch den Arzt ein solches Training zu Hause veranlasst wird, sollte insbesondere die Bereitschaft zum Training und zur Kommunikation überprüft und die absoluten und relativen Kontraindikationen für kardiopulmonale Belastungen besprochen werden [11]. Es sollte betont werden, dass es sich um Ergebnisse einer Pilotuntersuchung handelt, sodass weitere Untersuchungen in größeren Kollektiven zur Evaluation der Praxistauglichkeit erforderlich sind.

\section{Bedeutung und Ziel des körperlichen Trainings \\ $\nabla$}

Bei der Therapie der chronisch obstruktiven Lungenerkrankung (COPD) wird neben der Verbesserung der Obstruktion und Verminderung der Symptome die Verbesserung der körperlichen Leistungsfähigkeit in den GOLD-Empfehlungen vor allem durch ein Ergometertraining der unteren Extremitäten angestrebt [12]. Das Training der unteren Extremitäten führt im Vergleich zu Armtraining zu einer wesentlich relevanteren Leistungsverbesserung. Für das Beintraining setzten wir das Trainingsgerät nicht ein, da eine stabilere bzw. standfestere Version des Minitrimmers erforderlich sein dürfte. Die Patienten trainierten deshalb in einer zeitlich getrennten Sitzung auf einem Fahrradergometer. Im Vergleich zu gesunden Personen ist die Muskelkraft der unteren Extremitäten bei COPD deutlich vermindert. So ist zum Beispiel der Querschnitt der Oberschenkelmuskulatur im Vergleich zu Gesunden von 110 auf $83 \mathrm{~cm}^{2}$ reduziert [13]. Bezieht man die Muskelkraft auf den Muskelquerschnitt, besteht kein Unterschied zwischen Gesunden und COPD-Patienten, sodass die Herabsetzung der Muskelkraft auf eine Muskelatrophie zurückzuführen ist, deren Ursache unter anderem auf einer chronischen verminderten körperlichen Aktivität beruht.

Körperliche Aktivität korreliert direkt mit der Lebenserwartung [3]. In einer Studie mit 2386 Patienten mit COPD wurde über 20 Jahre hinweg die Lebenserwartung überprüft. Patienten mit höherer körperlicher Aktivität wiesen eine signifikant geringere Mortalität (Hazard Ratio, $\mathrm{HR}=0,70$ ) gegenüber Patienten mit sehr geringer körperlicher Aktivität auf. Patienten mit körperlicher Aktivität von $>4$ Stunden pro Woche erreichten die günstigsten Ergebnisse. Diese Patienten wurden auch seltener ins Krankenhaus eingewiesen ( $\mathrm{HR}=0,72)$. Deshalb sollten Patienten dazu ermutigt werden, ihre regelmäßige körperliche Aktivität zu erhöhen beziehungsweise zu erhalten.

Ein Rehabilitationsprogramm sollte sich auch positiv auf die körperliche Aktivität im täglichen Leben auswirken. In einer Studie an 29 Patienten mit COPD wurde nach drei und sechs Monaten ein Aktivitäts-Monitoring durchgeführt [14]. Das Rehabilitations-Programm bestand in den ersten drei Monaten aus einem multidisziplinären Trainingsprogramm jeweils dreimal pro Woche und in den letzten drei Monaten trainierten die Patienten zweimal pro Woche in einem Rehabilitationszentrum. Während sich schon nach drei Monaten die Bewegungsintensität, die Belastungskapazität, die Muskelkraft und Lebensqualität besserte, stieg die tägliche Bewegungszeit - gemessen mit einem Akzelerometer - nicht an. Nachdem allerdings die Rehabilitation weitere drei Monate durchgeführt worden war, stieg auch die tägliche Bewegungszeit signifikant um $20 \%$ an. Wenn man also die tägliche körperliche Aktivität bei COPD-Patienten steigern will, sind vor allem über mehrere Monate angelegte Trainingskonzepte 
sinnvoll. Allerdings gehen die gewonnenen vorteilhaften Effekte einer 6-12-wöchigen Rehabilitation auf Leistungsfähigkeit und Lebensqualität über 12-18 Monate fast wieder auf den Ausgangswert zurück [2].

\section{Regelmäßige Betreuung}

$\nabla$

Der Vorteil des telemonitorisch betreuten Trainings besteht in der Möglichkeit, auf der Grundlage der objektiven Trainingszeiten über Telefon den Patienten regelmäßig zu motivieren. Hausbesuche sind aufwendig und aus Kostengründen nur selten möglich, dagegen sind Telefonkontakte weniger aufwendig, aber die Information zur aktuellen Trainingsleistung ist nicht zuverlässig genug.

Die genannten Ergebnisse weisen darauf hin, dass eine kontinuierliche Betreuung und Motivation des Patienten sinnvoll ist. Bei 21 Patienten mit schwergradiger COPD wurde nach Ermittlung der Basisdaten über 14 Tage hinweg jeden 2. Tag ein Telefonanruf getätigt mit dem Ziel, die tägliche körperliche Aktivität auf $3 \times 15$ min bei $75 \%$ der maximalen Dyspnoe zu erhöhen [4]. Verglichen mit den Basisdaten konnte die mit einem Pedometer und einem Aktigraphen gemessene körperliche Aktivität und die 6-Minuten-Gehstrecke gesteigert werden. Die Indizes für Lebensqualität (SGRQ und SF-36) zeigten ebenfalls eine signifikante Verbesserung. Diese Untersuchung bestätigt damit, dass zumindest kurzfristig durch einfache Intervention wie kurzer Telefonkontakt die Motivation und damit die Leistung selbst ohne vorangehende Rehabilitationsphase erhöht werden kann.

Wenn eine nachhaltige Verbesserung der körperlichen Aktivität erzielt werden soll, empfiehlt es sich, die Verordnung von Training mit regelmäßiger Beratung, basierend auf Theorien der Verhaltensänderung zu kombinieren [15].

Schlussfolgernd dürfte demnach das häusliche Ergometer-Training in der Kombination mit der Online-Übertragung (Telemonitoring) und regelmäßiger Telefonbetreuung ein kostengünstiges Verfahren zur Erhöhung der körperlichen Aktivität darstellen. Mit relativ geringen Kosten kann die Trainingszeit zu Hause am Fahrrad- und Arm-Ergometer telemonitorisch durch geschulte Fachkräfte überwacht werden. Die individualisierte Betreuung ermöglicht eine Steigerung der Motivation zum Training. Durch Anbindung an ein E-health-Center, an die Klinik oder an den be- treuenden Hausarzt erhält der Patient ständige Rückmeldung, sodass gute Chancen bestehen, dass die Trainingsmotivation erhalten bleibt.

\section{Literatur}

1 Hui KP, Hewitt $A B$. A simple pulmonary rehabilitation program improves health outcomes and reduces hospital utilization in patients with COPD. Chest 2003; 124: 94-97

2 Ries AL, Kaplan RM, Myers R et al. Maintenance after pulmonary rehabilitation in chronic lung disease: a randomized trial. Am J Respir Crit Care Med 2003; 167: 880-888

3 Garcia-Aymerich J, Lange P, Benet $M$ et al. Regular physical activity reduces hospital admission and mortality in chronic obstructive pulmonary disease: a population based cohort study. Thorax 2006; 61: $772-$ 778

4 Wewel AR, Gellermann I, Schwertfeger I et al. Intervention by phone calls raises domiciliary activity and exercise capacity in patients with severe COPD. Respir Med 2008; 102: 20-26

5 Kamps A, Schüle K. Zyklisches Bewegungstraining der unteren Extremitäten in der Schlaganfallrehabilitation. Neurol Rehabil 2005; 11: 1 12

6 Godin G, Shephard RJ. A simple method to assess exercise behavior in the community. Can J Appl Sport Sci 1985; 10: 141 - 146

7 Marcus BH, Selby VC, Niaura RS et al. Self-efficacy and the stages of exercise behavior change. Research Quarterly for Exercise and Sport 1992; 63: 60-66

8 Gigliotti F, Coli C, Bianchi R et al. Arm exercise and hyperinflation in patients with COPD: effect of arm training. Chest 2005; 128: 1225-1232

9 Ries AL, Bauldoff GS, Carlin BW et al. Pulmonary Rehabilitation: Joint ACCP/AACVPR Evidence-Based Clinical Practice Guidelines. Chest 2007; $131: 4 S-42 S$

10 Hillsdon M, Foster C, Thorogood M. Interventions for promoting physical activity. Cochrane Database Syst Rev 2005; 1: CD003180

11 Rühle $\mathrm{KH}$. Praxisleitfaden der Spiroergometrie. 2. Auflage. Stuttgart: Kohlhammer, 2008

12 Rabe KF, Hurd S, Anzueto A et al. Global strategy for the diagnosis, management, and prevention of chronic obstructive pulmonary disease: GOLD Executive Summary. Am J Respir Crit Care Med 2007; 176: $532-555$

13 Bernard S, LeBlanc P, Whittom F et al. Peripheral muscle weakness in patients with chronic obstructive pulmonary disease. Am J Respir Crit Care Med 1998; 158: 629-634

14 Pitta F, Troosters T, Probst VS et al. Are patients with COPD more active after pulmonary rehabilitation? Chest 2008; 134: 273-280

15 Little P, Dorward M, Gralton $S$ et al. A randomised controlled trial of three pragmatic approaches to initiate increased physical activity in sedentary patients with risk factors for cardiovascular disease. Br J Gen Pract 2004; 54: 189-195 